\title{
Slip lines in front of a round notch tip in a pressure-sensitive material
}

\author{
H.-Y. Jeong ${ }^{\text {a }}$, X.-W. Li ${ }^{\text {b }}$, A. F. Yee ${ }^{\text {b }}$, J. Pan ${ }^{\text {a }}$ \\ ${ }^{a}$ Mechanical Engineering and Applied Mechanics, The University of Michigan, Ann Arbor, MI 48109, USA \\ ${ }^{b}$ Material Science and Engineering, The University of Michigan, Ann Arbor, MI 48109, USA
}

Received 15 April 1993; revised version received 2 November 1993

\begin{abstract}
Plastic deformation in the form of slip lines is observed in front of notches in pure epoxy and rubber-toughened epoxy specimens under four-point bending. In contrast to the case for pressure-insensitive materials, the angles between two families of slip lines emanating from notch surfaces are not $90^{\circ}$ : they are smaller than $90^{\circ}$ under tension, but they are larger than $90^{\circ}$ under compression. For the pure epoxy specimen, the angles are almost constant throughout plastic zones, and the deviations of the angles from $90^{\circ}$ are almost the same under both tension and compression. For the rubber-toughened epoxy specimen, however, the angles are not constant throughout the plastic zone under tension although they are almost constant under compression. In addition, the angles for the rubber-toughened specimen under tension are smaller than those for the pure epoxy specimen under tension, but the angles for the rubber-toughened specimen under compression are larger than those for the pure epoxy specimen under compression. Furthermore, theoretical slip lines are constructed around notches for pressure-sensitive perfectly plastic materials. These slip lines are in good agreement with the slip lines observed in the pure epoxy specimen.
\end{abstract}

\section{Introduction}

In classical plasticity theories, it is generally assumed that plastic yielding is independent of the hydrostatic stress and plastic dilatancy is negligible. Although these are good assumptions for metallic materials without voids or weakly bonded inclusions (Spitzig et al., 1975, 1976), pressure-sensitive yielding and plastic dilatancy cannot be ignored in many materials such as rocks (Rudnicki and Rice, 1975), soils and polymers (Sternstein and Ongchin, 1969; Spitzig and Richmond, 1979; Kinloch and Young, 1983). In zirconia-containing ceramics, the tetragonal-to-monoclinic phase transformation is also pressure-sensitive, and the corresponding volume increase is an important source of toughening for the ceramics, see, for example, McMeeking and Evans
(1982) and Budianski et al. (1983). As for plastic flow, the normality flow rule is not always valid for pressure-sensitive materials. For example, experiments on polyethylene and polycarbonate have shown much less plastic volume increases than those predicted by the normality flow rule (Spitzig and Richmond, 1979). Therefore, in order to understand the plastic flow and the subsequent fracture of the materials, pressure-sensitive yielding or phase transformation and plastic dilatancy, as determined by the normality or non-normality flow rule, must be taken into account.

Several slip-line studies related to cracks have been carried out based on the pressure-insensitive von Mises yield criterion and plastic incompressibility. For rigid perfectly plastic von Mises materials, Rice (1968) proposed the well-known Prandtl field around a sharp 
crack tip and showed intense straining above and below the crack tip. Since intense straining ahead of a crack tip is required for ductile fracture, its absence resulting from the Prandtl field seems to be incorrect for modelling ductile fracture. Rice and Johnson (1970) resolved this paradox by considering the large geometry change of a sharp crack tip by blunting. They obtained a slip-line field near a blunted crack tip and demonstrated intense straining directly ahead of the crack tip based on the slip-line field. McMeeking (1977b) confirmed the result of Rice and Johnson (1970) through finite element computations. McMeeking (1977a) also showed slip-line fields for three differently blunted crack-tip shapes.

Toughening of epoxy resins by the addition of rubber particles has been proven to be effective. Yee and his co-worker Pearson (Yee and Pearson, 1986; Pearson and Yee, 1986) suggested that the sources of toughness in such materials are the cavitation occurring inside the rubber particles and the subsequent plastic shear yielding in the epoxy matrix. In order to understand these phenomena, we conducted fourpoint bending tests of double-notched pure epoxy and rubber-toughened epoxy specimens, and studied the slip-line fields in detail. In parallel with the experimental investigation, we also carried out a theoretical investigation. In the latter, we adopted Coulomb's yield criterion and a plastic potential function similar to the yield criterion to account for pressure-sensitive yielding with normality or non-normality flow, and then constructed theoretical slip-line fields near round notch tips with the assumption of rigid perfectly plastic material behavior. These theoretical slip-line fields were compared with the experimental observations of the slip-line fields near the round notch tips under tension and compression.

\section{Experiments}

In order to characterize possible deformation patterns near sharp crack tips, we conducted four-point bending tests of double-notched pure epoxy and rubber-toughened epoxy specimens, as shown in Fig. 1. The pure epoxy consists of 100 parts of DER ${ }^{R}$ 331 and 5 parts of piperidine. The rubber-toughened epoxy consists of 100 parts of $D^{2} R^{R} 331,5$ parts of piperidine and 2.5 parts of $\mathrm{HYCAR}^{\mathrm{R}} \mathrm{CTB} 2000 \mathrm{X} 162$

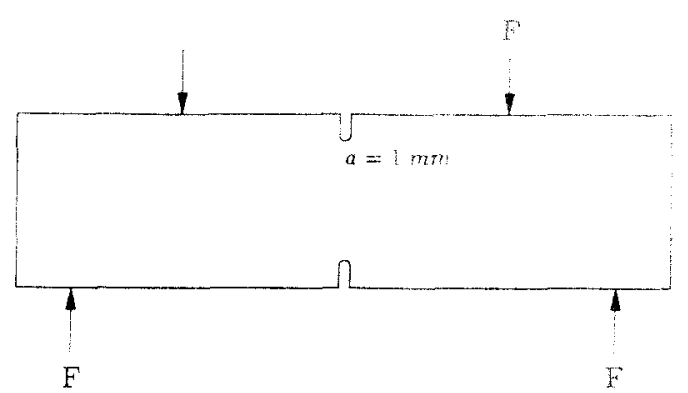

Fig. 1. A double-notched specimen under four-point bending.

rubber. $D^{2} R^{R} 331$ is a liquid diglycidyl ether of bisphenol-A (DGEBA) epoxy and HYCAR ${ }^{R}$ CTB $2000 \times 162$ is a carboxyl-terminated butadiene homopolymer. The pure epoxy and rubber-toughened epoxy were cured at $120^{\circ} \mathrm{C}$ for 16 hours, and the preparation of these epoxies is described in Pearson and Yee (1991). The mechanical properties of the pure and rubber-toughened epoxies are listed in Table 1. The mechanical properties were obtained by tension and compression tests conducted at a displacement rate of $0.1 \mathrm{in} / \mathrm{min}$. The notch tip radii of the specimens were $1 \mathrm{~mm}$, and the bending tests were conducted at a displacement rate of $0.1 \mathrm{in} / \mathrm{min}$. It should be noted that if a sharp crack tip indeed becomes blunted due to plastic yielding under Mode I plane strain loading, the estimated root radius at initiation of crack growth or fracture is in the order of $\mu m$ based on the value of $K_{\text {Ic }}^{2} / E \sigma_{\mathrm{t}}$. Here, the critical stress intensity factor $K_{\text {Ic }}$ for pure epoxies is taken as $0.8 \mathrm{MPa} \mathrm{m}^{1 / 2}$ (Pearson and Yee, 1991), Young's modulus $E$ is $3.0 \mathrm{GPa}$ and the tensile yield stress $\sigma_{\mathrm{t}}$ is $74 \mathrm{MPa}$. Since it was not easy to observe the ma-

Table 1

Mechanical properties of pure and rubber-toughened epoxies

\begin{tabular}{lll}
\hline & Material & \\
\cline { 2 - 3 } & Pure epoxy & $\begin{array}{l}\text { Rubber-toughened } \\
\text { epoxy }\end{array}$ \\
\hline $\begin{array}{l}\text { Young's modulus } \\
\begin{array}{l}E(\mathrm{GPa}) \\
\text { Tensile yield stress } \\
\sigma_{\mathrm{I}}(\mathrm{MPa})\end{array}\end{array}$ & 3.0 & 2.8 \\
$\begin{array}{l}\text { Compressive yield } \\
\text { stress } \sigma_{\mathrm{c}}(\mathrm{MPa})\end{array}$ & 86 & 71 \\
\hline
\end{tabular}


terial behavior in front of a sharp crack tip, we used specimens with notches of comparatively large radii.

Slip-line fields near the notch tips under tension and compression were observed and photographed through an optical microscope equipped with polarizers. For the pure epoxy specimen, Fig. 2(a) shows the slipline field under tension and Fig. 2(b) the slip-line field under compression. For the rubber-toughened epoxy specimen, Fig. 3(a) shows the slip-line field under tension and Fig. 3(b) the slip-line field under compression. For both these specimens, the angles between the two families of slip lines emanating from notch surfaces are less than $90^{\circ}$ under tension, but more than $90^{\circ}$ under compression. For the pure epoxy specimen, the experimental results show that the angles are almost constant throughout the plastic zones, and the deviations of the angles from $90^{\circ}$ are almost the same in magnitude under tension and compression although the signs are opposite. The average deviation is $6.86^{\circ}$. In addition, the plastic zone under tension is larger than that under compression.

For the rubber-toughened epoxy specimen, the experimental results show that by the addition of rubber particles, the angles between the two families of slip lines become smaller under tension, but larger under compression, when compared to those of the pure epoxy specimen. In this case, the deviation of the angles from $90^{\circ}$ is not constant under tension, and it increases with the distance from the notch tip. However, the deviation of the angles is almost constant with respect to the distance from the notch tip under compression. It is also important to note that in the slip-line field produced by tension, the rubber particles are cavitated, i.e., small voids have formed and grown in the rubber particles. Each cavitated rubber particle behaves as a small stress concentrator and as a source of new slip lines which form around it during loading. This changes both the orientation and density of slip lines. These newly created slip lines tend to be well-distributed and lie throughout many layers of the matrix, making the matrix appear more damaged. The dispersed slip-line field contributes to the ability of the rubber-toughened epoxy to dissipate more energy by plastic deformation and thus improves its fracture toughness. A detailed study of the influence of the rubber particle size and particle size distribution on deformation patterns near notch tips and on toughening mechanisms in rubber-modified epoxies can be found in Pearson (1990) and Pearson and Yee (1991). The influence of the volume fraction of the rubber particle on the deformation patterns, fracture mechanisms and constraint conditions ahead of notch tips can be found in Yee et al. (1993).

\section{Theoretical approach}

\subsection{Coulomb's yield criterion}

In order to explain the two different slip-line patterns observed in the pure epoxy specimen under tension and compression, we employ a pressuresensitive yield criterion, where yielding is dependent on a linear combination of the effective shear stress $\tau_{\mathrm{e}}$ and the hydrostatic stress $\sigma_{m}$. The pressure-sensitive Coulomb yield criterion (Drucker and Prager, 1952) is expressed as

$f(\boldsymbol{\sigma})=\tau_{\mathrm{e}}+\mu \sigma_{m}=\tau_{0}$,

where

$\tau_{\mathrm{e}}=\left(\frac{\sigma_{i j}^{\prime} \sigma_{i j}^{\prime}}{2}\right)^{1 / 2}, \quad \sigma_{i j}^{\prime}=\sigma_{i j}-\sigma_{m} \delta_{i j}$,
$\sigma_{m}=\frac{\sigma_{k k}}{3}$

In Eqs. (1) and (2), $\sigma$ is the Cauchy stress tensor, $\mu$ is the pressure-sensitivity factor, $\tau_{0}$ is the shear yield stress, and $\delta_{i j}$ is the Kronecker delta. Indices $i, j$ and $k$ range from 1 to 3 , and summation convention applies for repeated indices. Note that Coulomb's yield criterion in Eq. (1) reduces to the von Mises yield criterion when $\mu$ becomes zero. As shown in Figs. 2 and 3 , the plastic deformation patterns resemble the classical slip-line patterns. Therefore, in this theoretical approach, we consider only the cases where plastic strains are much larger than elastic strains. We assume rigid perfectly plastic material behavior in order to take advantage of the classical slip-line theory.

The pressure-sensitivity factor $\mu$ can be obtained from the tensile yield stress $\sigma_{\mathrm{t}}$ and the compressive yield stress $\sigma_{\mathrm{c}}$ through the relation given in Needleman and Rice (1978):

$\mu=\sqrt{3} \frac{\sigma_{\mathrm{c}}-\sigma_{\mathrm{t}}}{\sigma_{\mathrm{c}}+\sigma_{\mathrm{t}}}$ 

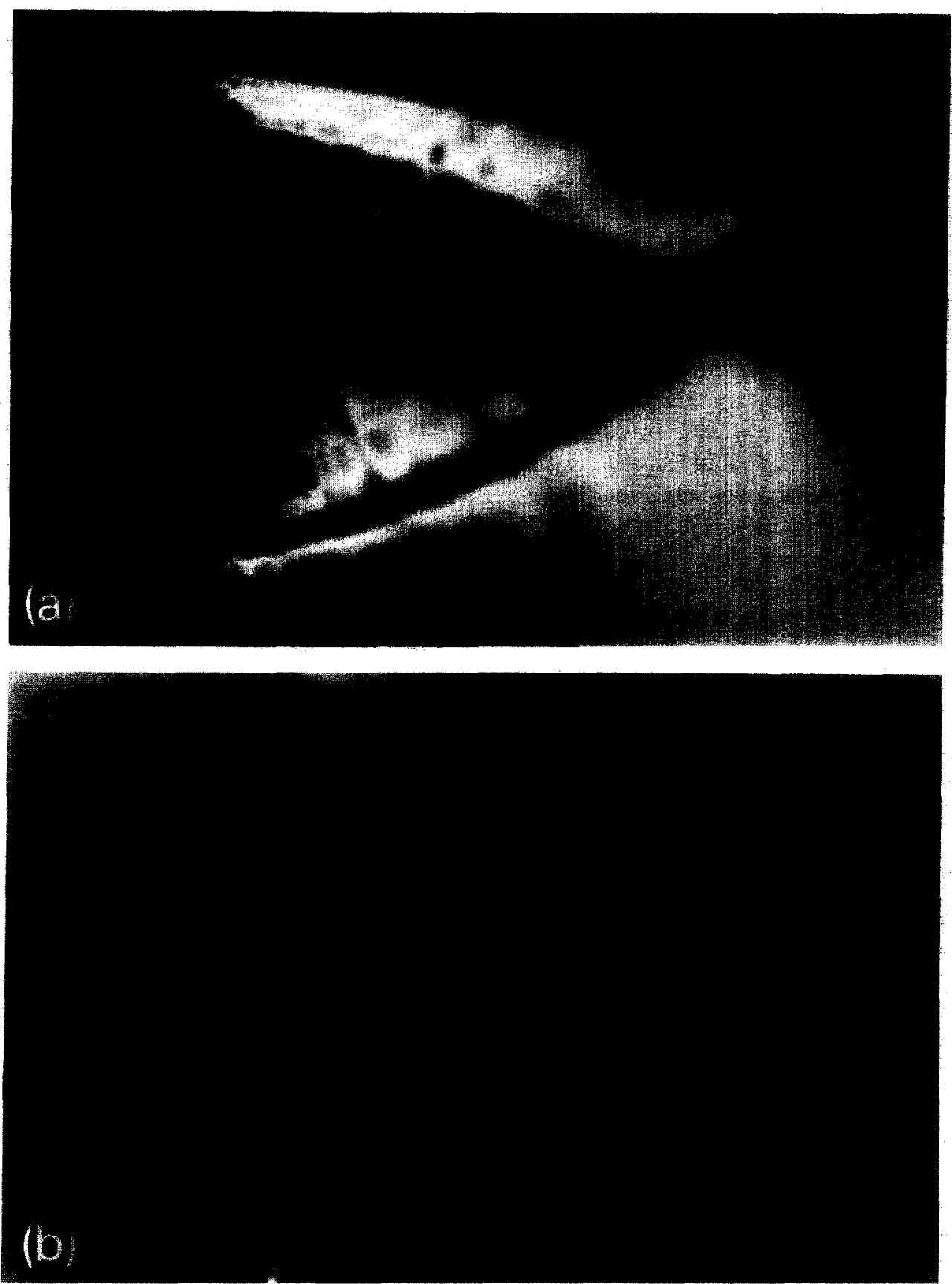

Fig. 2. Slip-line fields in front of notch tips in a pure epoxy specimen: (a) under tension; (b) under compression 

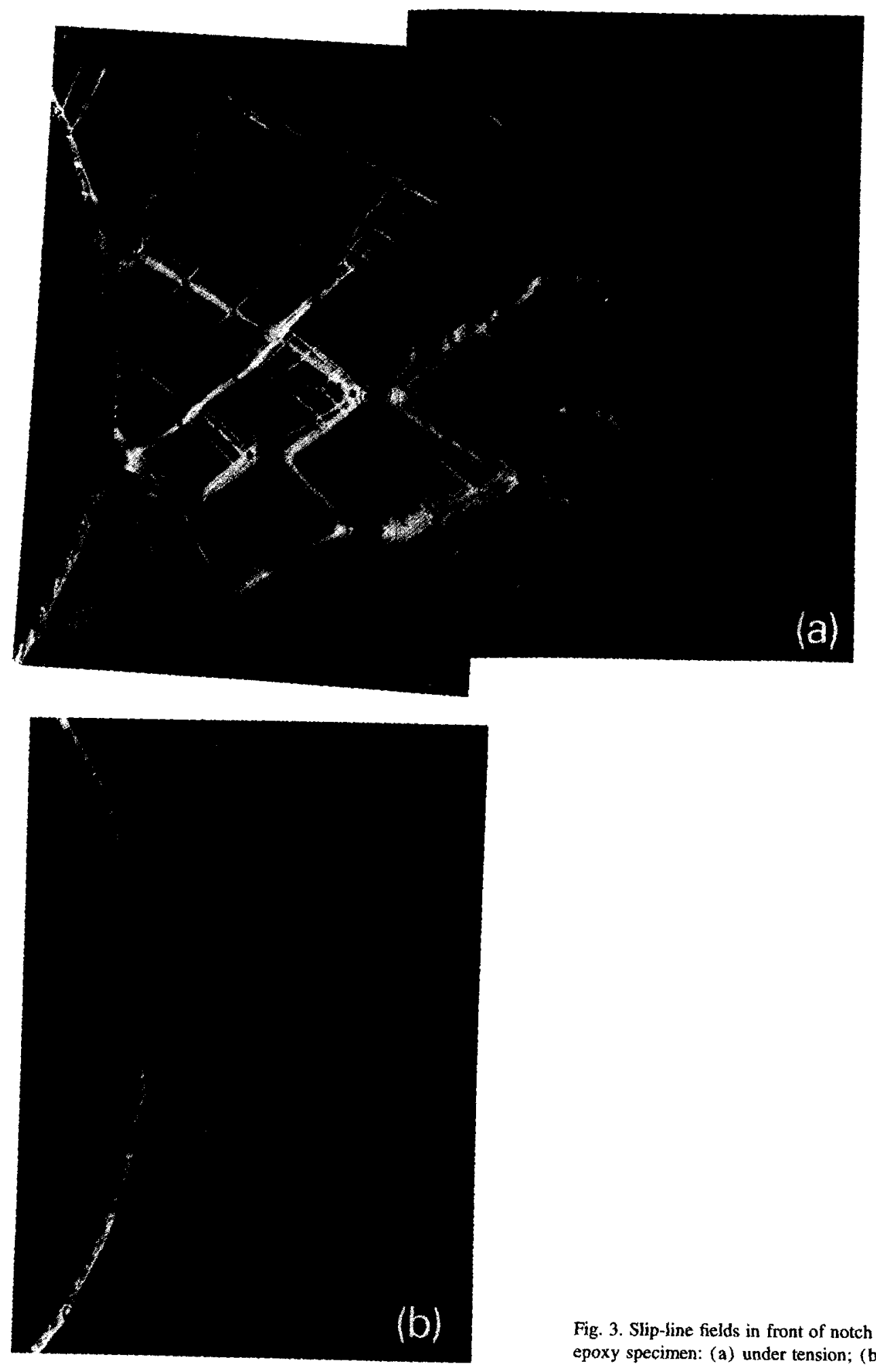

Fig. 3. Slip-line fields in front of notch tips in a rubber-toughened epoxy specimen: (a) under tension; (b) under compression. 
For polymers, Kinloch and Young (1983) reported that the values of $\mu$ lie between 0.10 and 0.25 . In passing, for several steels (HY-80, maraging, 4310 and 4330 steels), Spitzig et al. $(1975,1976)$ showed that the values of $\mu$ lie between 0.014 and 0.064 . For zirconia-containing ceramics, Coulomb's yield criterion can be used to characterize the tetragonal-tomonoclinic phase transformation. Chen (1991) reported that the values of $\mu$ for the phase transformation are 0.55 and 0.77 for $\mathrm{MgO}-\mathrm{PSZ}$ and $\mathrm{CeO}_{2}-\mathrm{TZP}$, respectively. It should also be noted that Yu and Shetty (1989) reported $\mu=0.93$ for $\mathrm{CeO}_{2}-\mathrm{TZP}$.

In order to account for non-normality flow; a plastic potential function similar to Coulomb's yield criterion is defined as (Rudnicki and Rice, 1975)

$f_{p}(\boldsymbol{\sigma})=\tau_{\mathrm{e}}+\beta \sigma_{m}=\tau_{p}$,

where $\beta$ is the plastic dilatancy factor. The plastic strain increments $\mathrm{d} \varepsilon_{i j}$ are then represented as

$\mathrm{d} \varepsilon_{i j}=\mathrm{d} \lambda \frac{\partial f_{p}}{\partial \sigma_{i j}}=\mathrm{d} \lambda\left(\frac{\sigma_{i j}^{\prime}}{2 \tau_{\mathrm{e}}}+\frac{\beta}{3} \delta_{i j}\right)$,

where $\mathrm{d} \lambda$ is a proportionality factor. Eq. (5) gives a relation between the plastic volumetric strain increment $\mathrm{d} \varepsilon_{k k}$ and the effective shear plastic strain increment $\mathrm{d} \gamma_{\mathrm{e}}$, as

$\mathrm{d} \varepsilon_{k k}=\beta \mathrm{d} \gamma_{\mathrm{e}}$

Here, $\mathrm{d} \gamma_{\mathrm{e}}$ is defined as $\left(2 \mathrm{~d} \varepsilon_{i j}^{\prime} \mathrm{d} \varepsilon_{i j}^{\prime}\right)^{1 / 2}$ where $\mathrm{d} \varepsilon_{i j}^{\prime}(=$ $\left.\mathrm{d} \varepsilon_{i j}-\mathrm{d} \varepsilon_{k k} \delta_{i j} / 3\right)$ represent the deviatoric plastic strain increments. For incompressible plastic flow, $\beta=0$. However, for normality flow, $\beta=\mu$.

From Eq. (5) with $\mathrm{d} \varepsilon_{33}=0$, the out-of-plane normal stress $\sigma_{33}$ can be expressed in terms of the inplane stresses $\sigma_{11}, \sigma_{22}$ and $\sigma_{12}$ as

$$
\begin{aligned}
\sigma_{33} & =\frac{\sigma_{11}+\sigma_{22}}{2} \\
- & \frac{\beta}{\sqrt{1-\beta^{2} / 3}}\left[\left(\frac{\sigma_{11}-\sigma_{22}}{2}\right)^{2}+\sigma_{12}\right]^{1 / 2}
\end{aligned}
$$

By substituting $\sigma_{33}$ into Eq. (1), Coulomb's yield criterion under plane strain conditions becomes

$$
\begin{gathered}
f(\boldsymbol{\sigma})=\frac{1-\mu \beta / 3}{\sqrt{1-\beta^{2} / 3}}\left[\left(\frac{\sigma_{11}-\sigma_{22}}{2}\right)^{2}+\sigma_{12}^{2}\right]^{1 / 2} \\
\mu \frac{\sigma_{11}+\sigma_{22}}{2}=\tau_{0}
\end{gathered}
$$

For convenience, we can rewrite Eq. (8) in the polar coordinates with two new parameters, $\phi$ and $c$, as

$$
\begin{aligned}
& {\left[\left(\frac{\sigma_{r r}-\sigma_{\theta \theta}}{2}\right)^{2}+\sigma_{r \theta}^{2}\right]^{1 / 2}+\sin \phi \frac{\sigma_{r r}+\sigma_{\theta \theta}}{2}} \\
& \quad=c \cos \phi,
\end{aligned}
$$

where

$\sin \phi=\frac{\mu \sqrt{1-\beta^{2} / 3}}{1-\mu \beta / 3}$,

$c=\tau_{0}\left(\frac{1-\beta^{2} / 3}{1-\mu^{2}-2 \mu \beta / 3+4 \mu^{2} \beta^{2} / 9}\right)^{1 / 2}$.

Eqs. (8) and (10) are more general than their counterparts in Li and Pan (1990), where only normality flow is considered. These equations show that both the pressure-sensitivity factor $\mu$ and the plastic dilatancy factor $\beta$ can affect the plane strain yield criterion and consequently slip-line patterns. When both $\mu$ and $\beta$ are small, $\beta$ only has a second order effect on the orientation of slip lines through the $\sin ^{-1}$ function in $\mathrm{Eq}$. (10).

The yield criterion given in Eq. (9) is equivalent to the original form of Coulomb's yield criterion, which is given as

$$
\tau=c-\sigma \tan \phi .
$$

Here, $\tau$ and $\sigma$ are the shear stress and the normal stress on a slip plane, respectively, and $c$ and $\phi$ are frequently called the cohesion and the angle of internal friction, respectively. Two families of slip lines ( $\alpha$-lines and $\beta$-lines) satisfying Eq. (11) are inclined at an angle of $\pi / 4+\phi / 2$ from the major principal stress axis, as shown in Fig. 4. Note that the slip lines shown in Fig. 4 are not the lines of maximum shear stress except when $\mu$ (or $\phi$ ) is equal to zero.

\subsection{Theoretical slip lines}

For pressure-insensitive von Mises materials, the slip lines around a circular arc of radius $a$ are logarithmic spirals, see, for example, Kachanov (1974). The 


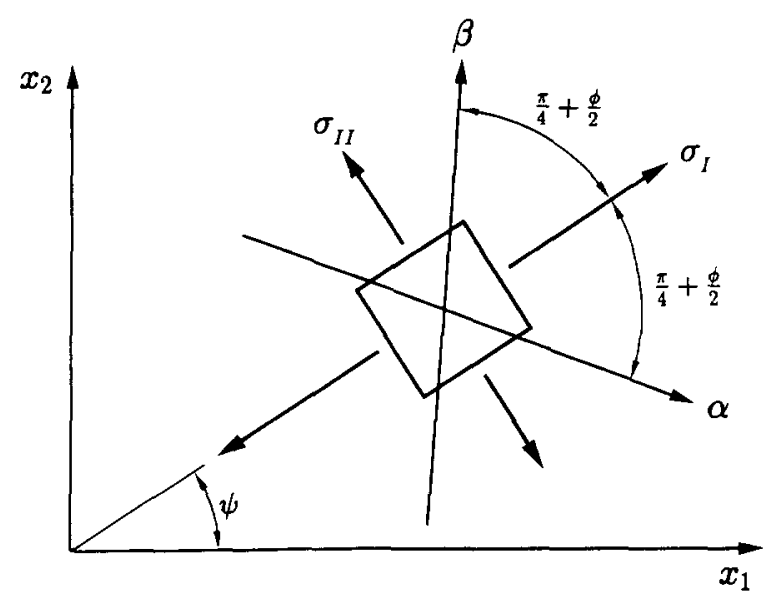

Fig. 4. Orientations of slip lines and the major and minor principal axes of stresses.

slip-line fields are the same under both positive and negative hydrostatic stresses, and they are represented as

$\theta-\ln \frac{r}{a}=$ const.

$\theta+\ln \frac{r}{a}=$ const.

For pressure-sensitive materials, however, the slip-line patterns become different according to the sign of the hydrostatic stress. Since slip lines for pressuresensitive materials are inclined at an angle of $\pi / 4+$ $\phi / 2$ from the major principal stress axis, the slip lines around a circular arc under a positive hydrostatic stress are represented as

$$
\begin{aligned}
& \left(\cos \frac{\phi}{2}+\sin \frac{\phi}{2}\right) \theta-\left(\cos \frac{\phi}{2}-\sin \frac{\phi}{2}\right) \ln \frac{r}{a} \\
& =\text { const. }(\alpha) \\
& \left(\cos \frac{\phi}{2}+\sin \frac{\phi}{2}\right) \theta+\left(\cos \frac{\phi}{2}-\sin \frac{\phi}{2}\right) \ln \frac{r}{a} \\
& =\text { const. }(\beta) .
\end{aligned}
$$

Under a negative hydrostatic stress, they are represented as

$$
\begin{aligned}
& \left(\cos \frac{\phi}{2}-\sin \frac{\phi}{2}\right) \theta+\left(\cos \frac{\phi}{2}+\sin \frac{\phi}{2}\right) \ln \frac{r}{a} \\
& =\text { const. }(\alpha) \\
& \left(\cos \frac{\phi}{2}-\sin \frac{\phi}{2}\right) \theta-\left(\cos \frac{\phi}{2}+\sin \frac{\phi}{2}\right) \ln \frac{r}{a} \\
& =\text { const. }(\beta) .
\end{aligned}
$$

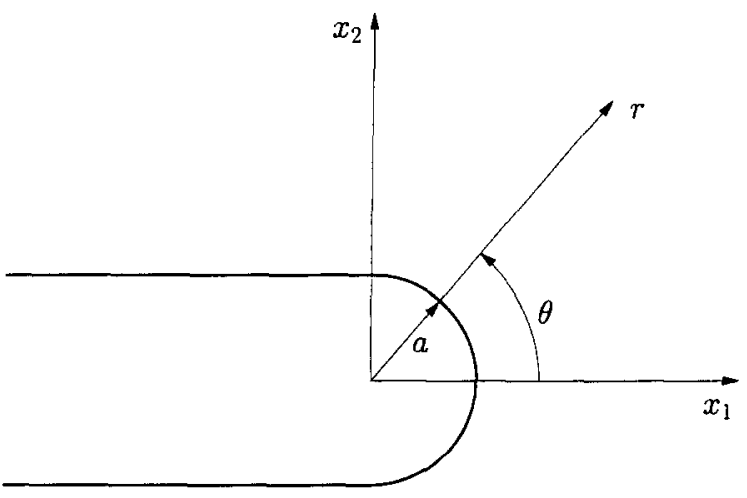

Fig. 5. Coordinate systems of a round notch tip.

These slip lines are the same as those emanating from the circular arc part of a round notch tip. A round notch tip in the polar and Cartesian coordinates is shown in Fig. 5.

Combining the yield criterion given in Eq. (11) and the equilibrium equations gives a further relationship between the normal stress $\sigma$ and the angle $\psi$ along slip lines ( $\mathrm{Li}$ and Pan, 1990)

$\chi-\psi=$ const. $(\alpha), \quad \chi+\psi=$ const. $(\beta)$,

where

$x=-\frac{1}{2 \tan \phi} \ln \left(1-\frac{\sigma}{c} \tan \phi\right)$

and $\psi$ is the angle between the $x_{1}$-axis and the major principal stress axis, as shown in Fig. 4.

Although the stress distributions in front of a notch tip can be obtained from Eq. (18), $\sigma_{r r}, \sigma_{\theta \theta}$ and $\sigma$ along the $x_{1}$-axis in plastic zones can also be calculated from the yield criterion given in Eq. (9) and the equilibrium equation in the $r$ direction. Under a positive hydrostatic stress, the stresses are given as

$$
\begin{aligned}
& \sigma_{r r}=\frac{q}{1-p}\left[1-\left(\frac{a}{r}\right)^{1-p}\right], \\
& \sigma_{\theta \theta}=\frac{p q}{1-p}\left[1-\left(\frac{a}{r}\right)^{1-p}\right]+q, \\
& \sigma=\frac{q}{2}\left\{\frac{1+p}{1-p}\left[1-\left(\frac{a}{r}\right)^{1-p}\right]+1\right\} .
\end{aligned}
$$

Under a negative hydrostatic stress, they are given as

$\sigma_{r r}=-\frac{q^{\prime}}{p^{\prime}-1}\left[\left(\frac{r}{a}\right)^{p^{\prime}-1}-1\right]$, 


$$
\begin{aligned}
& \sigma_{\theta \theta}=-\frac{p^{\prime} q^{\prime}}{p^{\prime}-1}\left[\left(\frac{r}{a}\right)^{p^{\prime}-1}-1\right]-q^{\prime}, \\
& \sigma=-\frac{q^{\prime}}{2}\left\{\frac{p^{\prime}+1}{p^{\prime}-1}\left[\left(\frac{r}{a}\right)^{p^{\prime}-1}-1\right]+1\right\},
\end{aligned}
$$

where

$p=\frac{1-\sin \phi}{1+\sin \phi}, \quad q=\frac{2 c \cos \phi}{1+\sin \phi}$,

$p^{\prime}=\frac{1}{p}, \quad q^{\prime}=\frac{q}{p}$

These stresses are valid up to

$r=a \exp \left\{\frac{\pi}{2} \frac{\cos (\phi / 2)+\sin (\phi / 2)}{\cos (\phi / 2)-\sin (\phi / 2)}\right\}$

under a positive hydrostatic stress and

$r=a \exp \left\{\frac{\pi}{2} \frac{\cos (\phi / 2)-\sin (\phi / 2)}{\cos (\phi / 2)+\sin (\phi / 2)}\right\}$

under a negative hydrostatic stress. For $\mu$ ( or $\phi$ ) equal to zero, the above-mentioned stresses become as follows:

$\sigma_{r r}= \pm 2 \tau_{0} \ln \frac{r}{a}$

$\sigma_{\theta \theta}= \pm 2 \tau_{0}\left(\ln \frac{r}{a}+1\right)$

$\sigma= \pm 2 \tau_{0}\left(\ln \frac{r}{a}+\frac{1}{2}\right)$

where the plus sign $(+)$ is for the case of a positive hydrostatic stress and the negative sign $(-)$ is for a negative hydrostatic stress. These stresses are valid up to $r=a \exp (\pi / 2)$. As expected, Eq. (18) is satisfied by the normal stress given in Eq. (21) or Eq. (24), and the corresponding slip lines given in Eqs. (14)(17).

\section{Discussion}

From Eq. (3) and the experimental data of tensile and compressive yield stresses, the pressure-sensitivity factor $\mu$ of the pure epoxy specimen is found to be 0.13 . Based on Eqs. (14)-(17), the fully developed slip-line fields for $\mu=\beta=0.13$ in front of round notch tips under tension and compression are shown
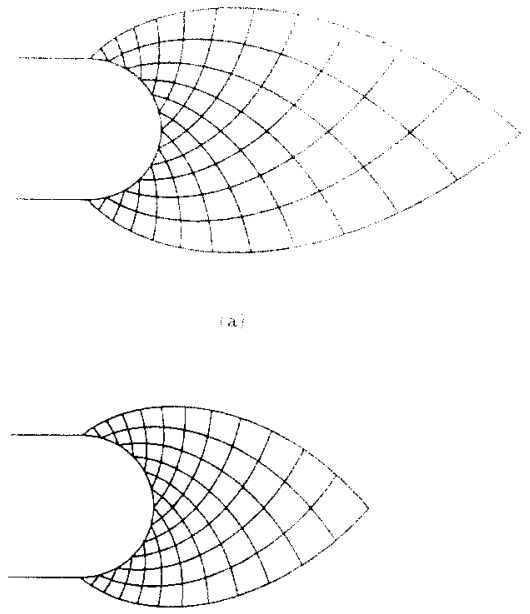

Fig. 6. Fully developed theoretical spiral slip-line fields in front of a round notch tip for $\mu=\beta=0.13$ : (a) under tension; (b) under compression.

in Figs. 6(a) and 6(b), respectively. In addition, the angle of deviation $\phi$ ( see Fig. 4) calculated from Eq. (10) is $7.49^{\circ}$ for the.normality flow of $\mu=\beta=0.13$ and $7.47^{\circ}$ for the non-normality flow of $\mu=0.13, \beta=$ 0 . This relative insensitivity of the angle $\phi$ to the value of $\beta$ indicates that the slip-line field for moderately pressure-sensitive materials hardly depends on the amount of plastic dilatancy, as discussed earlier for Eq. (10).

For the pure epoxy specimen, the theoretical slipline fields for $\mu=\beta=0.13$ shown in Fig. 6 are quite consistent with the experimental slip-line fields for pure epoxy shown in Fig. 2 in that the angles between two families of slip lines are less than $90^{\circ}$ under tension, but more than $90^{\circ}$ under compression, and the theoretical angle $\phi=7.49^{\circ}$ is close to the average angle $\phi=6.86^{\circ}$ observed in the experiments. Due to the relative brittleness of the pure epoxy specimen, the experimental slip-line fields shown in Fig. 2 are not as fully developed as those shown in Fig. 6.

As shown in Figs. 2 and 3, the angles between the two families of slip lines under tension are smaller for the rubber-toughened epoxy specimen than those for the pure epoxy specimen, but the angles under compression are larger for the rubber-toughened epoxy specimen than those for the pure epoxy specimen. This results from the additional macroscopic pressure- 


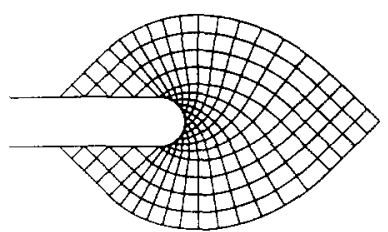

(a)

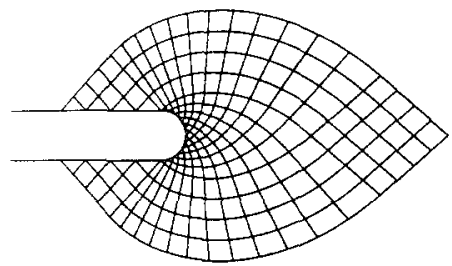

(b)

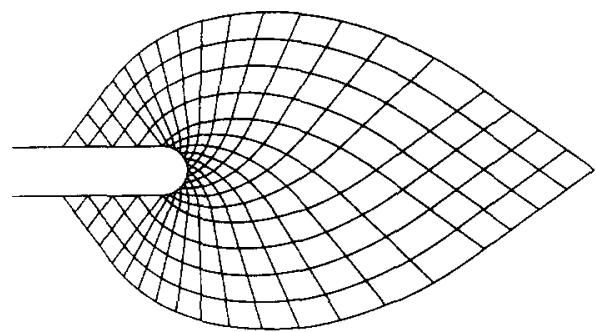

(c)

Fig. 7. Theoretical slip lines around a round notch tip under tension for: (a) $\mu=\beta=0.0$; (b) $\mu=\beta=0.13$; (c) $\mu=\beta=0.3$.

sensitive yielding due to the rubber particles in the rubber-toughened epoxy specimen. In contrast to the pure epoxy specimen, the angle $\phi$ for the rubbertoughened epoxy specimen increases with the distance from the notch tip under tension, but remains almost constant under compression. The variable angle $\phi$ under tension indicates that the macroscopic pressuresensitivity factor is not constant, which may be due to the increasing amount of cavitation and the interaction between rubber particles. In addition, for the tensile stress regions, the rubber-toughened epoxy specimen exhibits a significantly larger plastic zone than the pure epoxy specimen. For the compressive stress regions, however, the rubber-toughened epoxy specimen shows almost the same plastic zone size as the pure epoxy specimen. This observation can be easily explained by the incompressibility of rubber particles that do not contain voids.

For a sharp crack in either pure epoxies or rubber-

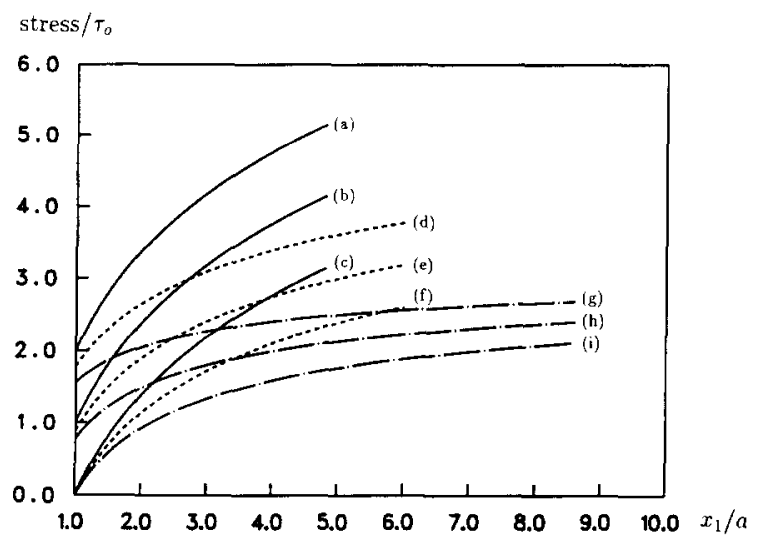

Fig. 8. Stress distributions ahead of a notch tip under tension for $\mu=\beta=0.0, \mu=\beta=0.13$ and $\mu=\beta=0.3$. (a) $\sigma_{\theta \theta} / \tau_{0}$ for $\mu=\beta=0$; (b) $\sigma / \tau_{0}$ for $\mu=\beta=0$; (c) $\sigma_{r r} / \tau_{0}$ for $\mu=\beta=0$; (d) $\sigma_{\theta \theta} / \tau_{0}$ for $\mu=\beta=0.13$; (e) $\sigma / \tau_{0}$ for $\mu=\beta=0.13$; (f) $\sigma_{r r} / \tau_{0}$ for $\mu=\beta=0.13$; (g) $\sigma_{\theta \theta} / \tau_{0}$ for $\mu=\beta=0.3$; (h) $\sigma / \tau_{0}$ for $\mu=\beta=0.3$; (i) $\sigma_{r r} / \tau_{0}$ for $\mu=\beta=0.3$.

toughened epoxies, a plastic deformation zone engulfs the crack tip. If the sharp crack tip indeed becomes blunted, the slip-line fields around the blunted tip can be obtained and are shown in Figs. 7 (a), (b) and (c) for $\mu=\beta=0, \mu=\beta=0.13$ and $\mu=\beta=$ 0.3 , respectively. The slip-line fields outside the spirals in front of the blunted notch tip approach the slipline fields for a sharp crack tip given by $\mathrm{Li}$ and Pan (1990). Furthermore, based on Eqs. (19)-(24), the stress distributions along the $x_{1}$-axis are also drawn in Fig. 8 for $\mu=\beta=0, \mu=\beta=0.13$ and $\mu=$ $\beta=0.3$. As Rice and Johnson (1970) pointed out, stresses are limited in front of a blunted crack tip, and the maximum stresses occur at the intersection point of the outmost spirals in front of the crack tip. The results shown in Fig. 8 also reveal the limited stresses in front of a round notch tip. In addition, the results show that as a material becomes more pressuresensitive, the stresses decrease.

It should be emphasized that the shear bands observed in experiments are assumed to be identified as the slip lines or the characteristics of the pressuresensitive yield function and the equilibrium equations with an assumption of rigid perfectly plastic material behavior under plane strain conditions. Eq. (10) indicates that for small values of $\mu$ and $\beta$, the orientation of the slip lines can be affected by the value of $\mu$ through the $\sin ^{-1}$ function. However, $\beta$ only has a second order effect on the orientation of the slip 
lines. There is another approach to examine the shear band formation, where the loss of ellipticity of the rate form of the governing field equations is sought for pressure-sensitive dilatant materials, see, for example, Rudnicki and Rice (1975) and Iwakuma and NematNasser (1982). Both research works show that, in addition to the pressure sensitivity and plastic dilatancy, the noncoaxiality of the stress rate and strain rate tensors can significantly affect the shear band formation and its orientation.

\section{Acknowledgement}

The support of this work from the Material Research Group grant funded by the National Science Foundation at The University of Michigan under grant no. DMR-8708405 is gratefully acknowledged. Helpful discussions with D.M. Li are also greatly appreciated.

\section{References}

Budiansky, B., J.W. Hutchinson and J.C. Lambropoulos (1983), Continuum theory of dilatant transformation toughening in ceramics, Int. J. Solids Structures 19, 337.

Chen, I.-W. (1991), Model of transformation toughening in brittle materials, J. Am. Ceram. Soc. 74, 2564.

Drucker, D.C. and W. Prager (1952), Soil mechanics and plastic analysis or limit design, Q. Appl. Math. 10, 157.

Iwakuma, T. and S. Nemat-Nasser (1982), An analytical estimate of shear band initiation in a necked bar, Int. J. Solids Structures $18,69$.

Kachanov, L.M. (1974), Fundamentals of the Theory of Plasticity, Mir Publishers, Moscow.

Kinloch, A.J. and R.J. Young (1983), Fracture Behavior of Polymers, Elsevier Applied Science, London.

Li, F.Z. and J. Pan (1990), Plane-strain crack-tip fields for pressure-sensitive dilatant materials, J. Appl. Mech. 57, 40.

McMeeking, R.M. (1977a), Blunting of a plane strain crack tip into a shape with vertices, Trans. ASME 99, 290.

McMeeking, R.M. (1977b), Finite deformation analysis of crack tip opening in elastic-plastic materials and implications for fracture, J. Mech. Phys. Solids 25, 357.

McMeeking, R.M. and A.G. Evans (1982), Mechanics of transformation-toughening in brittle materials, J. Am. Ceram. Soc. 65,242
Needleman, A. and J.R. Rice (1978), Limits to ductility set by plastic flow localization, in: D.P. Koistinen and N.M. Wang, ed., Mechanics of Sheet Metal Forming. Plenum Press, New York, p. 237.

Pearson, R.A. (1990), Sources of toughening in modified epoxies, Ph.D. Thesis, The University of Michigan, Ann Arbor, Michigan.

Pearson, R.A. and A.F. Yee (1986), Toughening mechanisms in elastomer-modified epoxies. Part 2: Microscopy studies, J. Mat. Sci. 2l, 2475.

Pearson, R.A. and A.F. Yee (1991), Influence of particle size and particle size distribution on toughening mechanisms in rubbermodified epoxies, J. Mat. Sci. 26, 3828.

Rice, J.R. (1968), A path independent integral and the approximate analysis of strain concentration by notches and cracks, J. Appl. Mech. 35. 379

Rice, J.R. and M.A. Johnson (1970), The role of large crack tip geometry changes in plane strain fracture, in: M.F. Kanninen, W.F. Adler, A.R. Rosenfield and R.I. Jaffee, eds., Inelastic Behavior of Solids, McGraw-Hill, New York, p. 641.

Rudnicki, J.W. and J.R. Rice (1975), Conditions for the localization of deformation in pressure-sensitive dilatant materials, J. Mech. Phys. Solids 23, 371 .

Spitzig. W.A., R.J. Sober and O. Richmond (1975), Pressure dependence of yielding and associated volume expansion in tempered martensite, Acta Metall. 23, 885.

Spitzig, W.A., R.J. Sober and O. Richmond (1976), The effect of hydrostatic pressure on the deformation behavior of maraging and HY-80 steels and its implications for plasticity theory, Metall. Trans. 7A, 1703.

Spitzig, W.A. and O. Richmond (1979), Effect of hydrostatic pressure on the deformation behavior of polyethylene and polycarbonate in tension and compression, Polym. Eng. Sci. 19, 1129.

Sternstein, S.S. and L. Ongchin (1969), Yield criteria for plastic deformation of glassy high polymers in general stress fields, Amer. Chem. Soc. Polym. Preprint 10, 1117.

Yee, A.F. and R.A. Pearson (1986), Toughening mechanisms in elastomer-modified epoxies. Part 1: Mechanical studies, $J$. Mat. Sci. 21,2462

Yee, A.F., D. Li and X. Li (1993), The important of constraint relief caused by rubber cavitation in the toughening of epoxy, J. Mat. Sci. 28,6392 .

Yu, C.-S. and D.K. Shetty (1989), Transformation zone shape, size, and crack-growth-resistance ( $R$-curve) behavior of ceriapartially-stabilized zirconia polycrystals, J. Am. Ceram. Soc. 72,921 . 